\title{
Formation by high power ultrasound of aggregated emulsions stabilised with milk protein concentrate (MPC70)
}

\author{
Ruijia Zhang ${ }^{\mathrm{a}}$, Lan Luo ${ }^{\mathrm{a}}$, Zhi Yang ${ }^{\mathrm{a}, *}$, Muthupandian Ashokkumar ${ }^{\mathrm{b}}$, Yacine Hemar ${ }^{\mathrm{c}, *}$ \\ ${ }^{a}$ School of Food and Advanced Technology, Massey University, Auckland 0632, New Zealand \\ ${ }^{\mathrm{b}}$ School of Chemistry, The University of Melbourne, Parkville, Victoria 3010, Australia \\ ${ }^{\mathrm{c}}$ Institute for Advanced Study, Shenzhen University, Shenzhen 518060, China
}

\section{A R T I C L E I N F O}

Given his role as Editor-in-Chief of this journal, Muthupandian Ashokkumar had no involvement in the peer-review of articles for which he was an author and had no access to information regarding their peer-review. Full responsibility for the peer-review process for this article was delegated to another Editor.

\section{Keywords:}

Emulsions

Milk protein concentrates

Microstructure

Viscosity

Oil droplets aggregation

\begin{abstract}
A B S T R A C T
In this work, oil-in-water emulsions stabilised by milk protein concentrate (MPC70) were investigated. The MPC70 concentration was kept constant at 5\% (close to the protein content found in skim milk) and the oil volume fraction was varied from 20 to $65 \%$. Sonication was performed at $20 \mathrm{kHz}$ and at a constant power of 14.4 W for a total emulsion volume of $10 \mathrm{~mL}$. Under certain oil concentration $(\geq 35 \%)$ and sonication times $(\geq 3 \mathrm{~s})$ the emulsion aggregated and formed high-viscosity pseudo plastic materials. However, the viscosity behaviour of the emulsion made with $35 \%$ oil reverted to that of a liquid if sonicated for longer times ( $\geq 15$ s). Confocal laser scanning microscopy showed clearly that the oil droplets are aggregated under the sonication conditions and oil concentrations indicated above. An attempt to explain this behaviour through a simple model based on the bridging of oil droplets by the MPC70 particles and, taking into account the oil droplet and MPC70 particle sizes as well as the oil volume fraction, was made. The model fails to describe in details the aggregation behaviour of these emulsions, likely due to the inhomogeneous protein layer, where both free caseins and casein micelles are adsorbed, and to the packing of the oil droplets at concentrations $\leq 55 \%$. Nonetheless, this work demonstrates the potential of ultrasound processing for the formation of dairy emulsions with tailored textures.
\end{abstract}

\section{Introduction}

The use of ultrasound (US) processing for emulsion preparation is extremely attractive since it allows obtaining nano-sized emulsions with narrow size distributions $[1,2]$. In addition, compared to conventional high pressure homogenisation (HPH) and other mixing methods, US is believed to require less energy $[3,4]$, while offering more flexibility in its implementation, i.e. it can be used in batch or continuous modes, and under temperature-controlled conditions. The efficiency of US for emulsion preparation is well documented and is attributed to US cavitation collapse which can generate considerable physical effects such as micro-jetting, micro-streaming, and shockwaves [5] leading to the breakup of the oil droplets and, in the presence of a surface-active agent, to the formation of a stable emulsion.

In recent years, the application of US to dairy ingredients and products has attracted a lot of interests due to its ability to improve their functional properties [6]. Of direct relevance to the present study, are studies on emulsions obtained by US and containing casein micelles- based dairy systems such as skim (SM) and whole milks (HM), skim milk powder (SMP), milk protein concentrates (MPC), and micellar casein powders (MCP). Casein micelle refers to the colloidal particle $\left(\sim 200 \mathrm{~nm}\right.$ in bovine milk) made of four different casein $\left(\alpha_{\mathrm{s} 1^{-}}, \alpha_{\mathrm{s} 2^{-}}, \beta-\right.$, and $\kappa$-casein) held together through electrical and hydrophobic interactions $[7,8]$ and containing colloidal calcium phosphate [9]. Almost all the studies found in the literature reported that the particle size of the emulsion made by the incorporation of oil into SM decreased with the sonication energy density, but did not result in a marked increase in viscosity [10-12]. This is expected, since the oil volume fraction $(\phi)$ used in these studies is relatively low $(\leq 10 \mathrm{v} / \mathrm{w})$. It was previously reported that HPH prepared emulsions stabilised by milk protein concentrate (MPC85) extensively aggregate when $\phi$ is higher than $25 \%$ [13]. In this short communication, we investigate soya oil emulsions stabilised by 5 wt $\%$ MPC70 with oil volume fraction $(\phi)$ varying between 20 and $60 \%$. MPC70 was chosen as its protein composition is similar to skim milk powder (SMP) but its lactose content is less than half that of SMP, and $5 \mathrm{wt} \%$ concentration is chosen to achieve a protein

\footnotetext{
* Corresponding authors.

E-mail addresses: Z.Yang2@massey.ac.nz (Z. Yang), yhemar@szu.edu.cn (Y. Hemar).
} 
concentration similar to that in SMP. Further, an advantage of MPC70 is that its solubility is known to be better than that of MPC85 which has a higher protein content [14]. We show that when $\phi \geq 35 \%$, the emulsion prepared by US treatment aggregates, but the extent of the aggregation depends on $\phi$. We discuss the findings of this study in the framework of protein particles bridging the oil droplets.

\section{Materials and methods}

\subsection{Materials and emulsion preparation}

MPC70 containing $69.9 \%$ proteins, $1.2 \%$ fat, $16.8 \%$ lactose, $4.7 \%$ moisture, and $7.4 \%$ ash was donated by Fonterra Co-operative Group Ltd. (Auckland, New Zealand). Soya oil was purchased from a New World supermarket in Auckland. Milli-Q water was used in all the samples preparation and all chemical reagents were purchased from Sigma-Aldrich (USA) and were of analytical grade.

To prepare the emulsions, first 5\% MPC70 powder was reconstituted in Milli-Q water containing $0.02 \mathrm{wt} \%$ sodium azide, as a preservative, under gentle magnetic stirring at room temperature for $2 \mathrm{~h}$. The MPC70 solution was then left overnight in a fridge at $4{ }^{\circ} \mathrm{C}$ to ensure full hydration. The next day, the MPC70 solution was first left to equilibrate to room temperature $\left(\sim 20^{\circ} \mathrm{C}\right)$ then the required amount of oil is added to the MPC70 solution to achieve the desired volume fraction $(\phi)$ and a total volume sample of $10 \mathrm{~mL}$, in a glass vial $(3 \mathrm{~cm}$ diameter and $7 \mathrm{~cm}$ height). The oil-MPC70 mixture was briefly pre-homogenised at room temperature using a rotor-stator homogeniser (T10 basic, IKA, Staufen, Germany) with a $10 \mathrm{~mm}$ probe (S10N-10G, IKA, Staufen, Germany) at $8000 \mathrm{rpm}$ for $7 \mathrm{~s}$. This pre-homogenised emulsion is then subjected to US treatments using a $20 \mathrm{kHz}$ ultrasound equipment (JY92-IIN, Ningbo Scientz Biotechnology Co.,Ltd) fitted with a $6 \mathrm{~mm}$ horn. The ultrasound treatment was performed at a constant power of $14.4 \mathrm{~W}$ determined by a calorimetric method [15] by measuring the increase in temperature of sonicated water with time. All emulsions were made at least in duplicates.

\subsection{Oil volume fraction and viscosity determinations}

The oil volume fraction is determined by weighing sample aliquots $(\sim 1.0 \mathrm{~g})$ in pre-weighed aluminium dishes. The dishes containing the sample were then placed in an oven (Labserv 8150, Longford, Ireland) preset at $105^{\circ} \mathrm{C}$ for $24 \mathrm{~h}$, after which they were weighed. The oil content is obtained by the substraction of the weight of the sample before and after drying. The oil volume fraction is then calculated by taking into account the soya oil density of $0.917 \mathrm{~g} / \mathrm{mL}$. Note that in the case of emulsions that are partially aggregated, to calculate the volume fraction of the aggregated phase the emulsion was first strained through a sieve with a mesh size of $\sim 1 \mathrm{~mm}$, and the retained phase was used in the measurements. All measurements were performed in duplicate.

The viscosity was measured using a stress-controlled rheometer (DHR-3, TA instruments, USA) using a parallel plate geometry $(40 \mathrm{~mm}$ in diameter, $1 \mathrm{~mm}$ gap). The measurement was performed in steadystate by applying a shear rate sweep from 0.1 to $1000 \mathrm{~s}^{-1}$ followed by a reverse sweep from 1000 to $0.1 \mathrm{~s}^{-1}$. These up-and-down measurements allow monitoring the extent of the emulsion aggregation which can be estimated by calculating the area $(S)$ of the hysteresis delimited by the up and down curves, using Origin Pro software (version 2021b, Origin Lab, MA, USA). All the measurements were performed in duplicate at a constant temperature of $20^{\circ} \mathrm{C}$.

\subsection{Particle size measurements and laser scanning confocal microscopy observation}

Before particle size measurement $1.0 \mathrm{~mL}$ emulsions were diluted in $1.0 \mathrm{~mL}$ of $1 \%$ SDS solution. This is done to ensure that all the aggregates are broken down [16] and that the MPC70 protein at the oil droplet interface is displaced, allowing to obtain the size of the core oil droplet diameter without its surrounding protein shell. The particle size distributions were determined using a Mastersizer 2000 (Malvern Instruments, Worcestershire, UK). Refractive indices of 1.47 and 1.33 were chosen for the soya oil and water [17]. All measurements were conducted in triplicates on duplicate samples at room temperature $\left(\sim 20^{\circ} \mathrm{C}\right)$.

Confocal laser scanning microscopy observations were performed on a Leica DM6000B microscope (Wetzlar, Germany). Fast green and Nile red were used to stain protein and oil phases, respectively. The stained emulsion samples were transferred onto concave microscopy slides and covered with a glass coverslip. Fast green and Nile Red were excited at the laser wavelength of $633 \mathrm{~nm}$ and $561 \mathrm{~nm}$, respectively.

\section{Results and discussion}

Under certain sonication conditions and oil volume fraction the
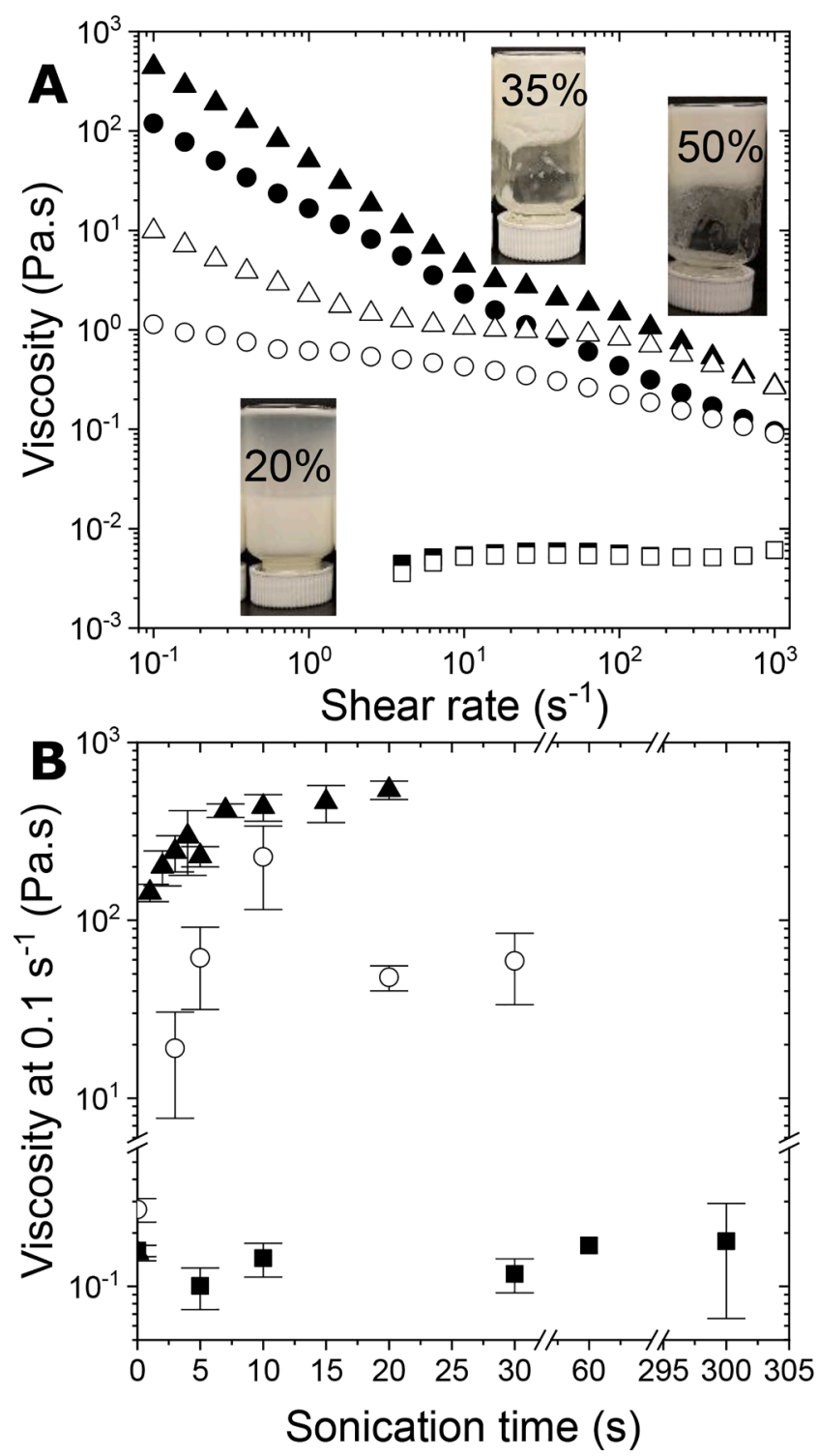

Fig. 1. (A) Viscosity as function of shear rate for emulsion containing $20 \%$ $(\square, \square), 35 \%(\bullet, \circ)$, and $50 \%(\Delta, \Delta)$ oil after 10 s US treatment. Solid and open symbols correspond to data obtained during the up and down measurements, respectively. Inset: photographs of the emulsions after $10 \mathrm{~s}$ sonication. (B) Viscosity at $0.1 \mathrm{~s}^{-1}$ as a function of US treatment time for emulsion made with

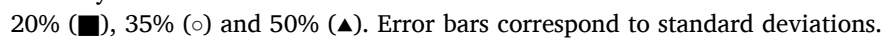


texture of the emulsion can vary from liquid to gelled. This is depicted in the photographs of the inverted vials containing the samples (Inset Fig. 1A), where emulsions made with 35 and $50 \%$ oil by sonication for $10 \mathrm{~s}$ adhere to the base of the vials, while emulsion made with $20 \%$ and sonicated for the same time flows. This can be also appreciated through the viscosity measurements where both the $35 \%$ and $50 \%$ emulsions show a steep pseudo-plastic behaviour, while the emulsion made with $20 \%$ oil remains Newtonian (Fig. 1A). The up (solid symbols) and down (open symbols) viscosity values as a function of shear rate show clearly that emulsions made with $35 \%$ and $50 \%$ are thixotropic, while the emulsion made with $20 \%$ is time-independent. This thixotropic behaviour will be exploited and discussed below when assessing the extent of emulsion aggregation.

In addition to the emulsion oil fraction, the extent of aggregation depends also on the sonication time (Fig. 1B). Before sonication, all the emulsions were liquid. The emulsion made with $20 \%$ oil remained liquid at all sonication times (even up to 5 min sonication, not shown in the Figure), while the emulsion made with $50 \%$ oil gelled within 3 s sonication and reached a plateau viscosity within the $10 \mathrm{~s}$ of sonication. The emulsion made with $35 \%$ forms a gel when sonicated between 3 and $10 \mathrm{~s}$ then reverts again to become liquid at longer sonication times. To monitor the extent of aggregation as a function of sonication time, the hysteresis area $(S)$ between the up and down curves of the viscosity as a function of shear rate was calculated. For emulsion made with $50 \%$ oil, the value of $S$ is $203 \pm 13,373 \pm 46$, and $413 \pm 68 \mathrm{~Pa}$, when sonicated for 3,10 and $15 \mathrm{~s}$, respectively; while at $35 \%$ the value of $S$ is $110 \pm 21$; $223 \pm 60$ and $50 \pm 2 \mathrm{~Pa}$, when sonicated for 5,10 , and 30 s, respectively. The high values of $S$ for the $50 \%$ emulsion is expected since $S$ has been suggested to be related to the energy required to breakup the aggregates [18-19] which depends on the number of oil droplets involved, which is higher at higher oil concentrations. Note that for non-sonicated emulsions and $20 \%$ sonicated emulsions, the value of $S$ was null since for these emulsions no hysteresis is observed.

To understand the mechanism behind the gelation/aggregation of these emulsions, confocal microscopy was used to observe their structures (Fig. 2). For all oil concentrations, prior to sonication, the prehomogenised emulsions were polydisperse with oil droplet size in the range of few $\mu \mathrm{m}$. Sonication reduced markedly the size of the oil droplets. From a structural viewpoint it can be seen that, in the case of emulsion made with $20 \%$ oil, the oil droplets are homogeneously distributed in the emulsion. However, in the case of the $35 \%$ emulsion, the structure of the emulsion is not homogeneous and does depend on the sonication time. At low sonication times ( $\leq 10 \mathrm{~s})$, large voids made mainly of water are entrapped between a large aggregate network containing both proteins and oil droplets. Then these voids disappear at higher sonication time, and the structure of the emulsion seems to become more homogeneous although some oil droplets and protein aggregates still can be seen. This change in the $35 \%$ emulsion explains the change in viscosity, as high sonication times result in the breakup of the droplets aggregates, resulting in turn in the decrease in viscosity (Fig. 1.B). At higher oil concentrations, voids can be also observed, however their size is much smaller than those observed at $35 \%$ oil concentration and further sonication does not get rid of these voids. This can be explained as follows, because of the marked increase in viscosity with sonication for the high concentrated emulsion sonication becomes ineffective since ultrasound cavitation is hindered by the high viscosity.

To analyse further the microstructure of the emulsions and to validate the confocal microscopy observations, particle-sizing using static light scattering were carried out (Fig. 3). Before sonication, the prehomogenised emulsions present quasi-monomodal particle size
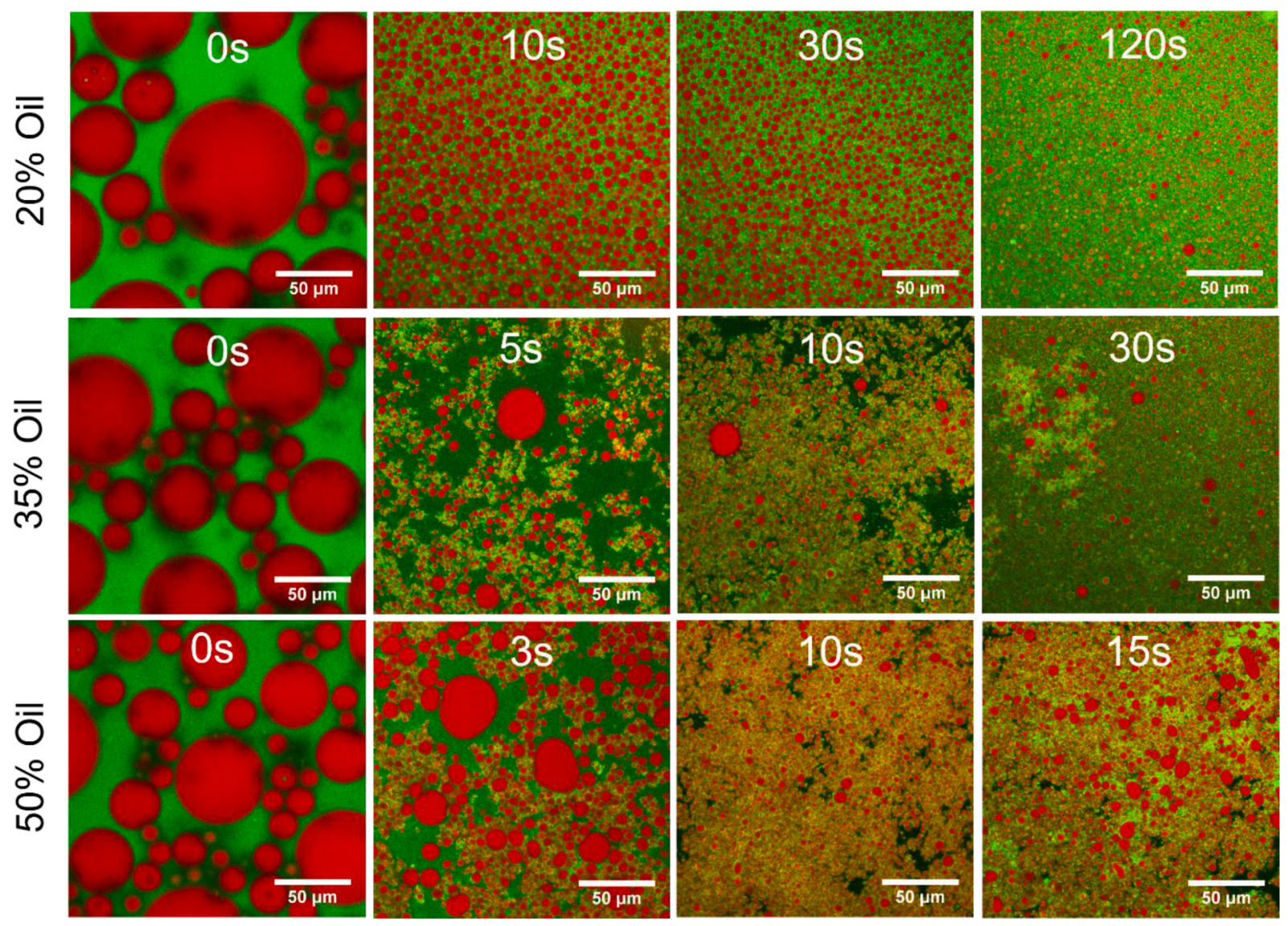

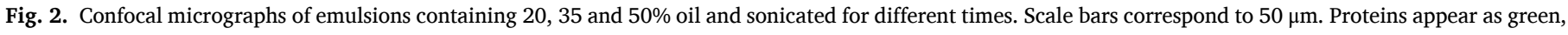
while the oil phase appears as red. 

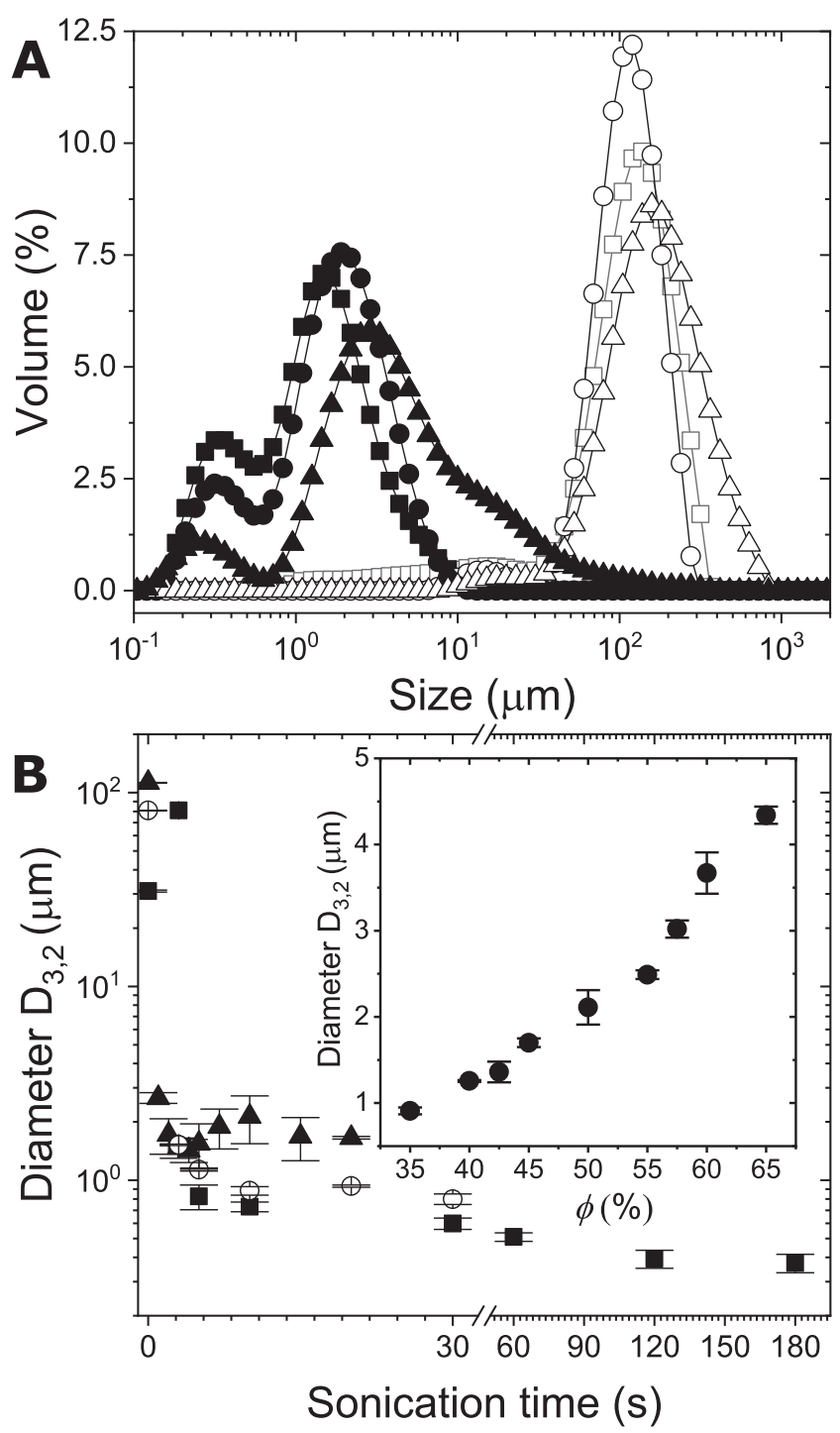

Fig. 3. (A) Particle size distributions of emulsion containing $20 \%(\square, \square), 35 \%$ $(\boldsymbol{\bullet}, \circ)$, and $50 \%(\boldsymbol{\Lambda}, \Delta)$. Open symbols are for non-sonicated emulsions and solid symbols are for emulsions sonicated for $10 \mathrm{~s}$. (B) Sauter mear diameter $\left(\mathrm{D}_{3,2}\right)$ as a function of sonication time for emulsion made with $20 \%(\square), 35 \%(0)$, and $50 \%(\Delta)$ oil. Inset: $\mathrm{D}_{3,2}$ at emulsion aggregation threshold as a function of the oil volume fraction $(\phi)$. Error bars correspond to standard deviations.

distributions ranging between $\sim 40$ to $\sim 800 \mu \mathrm{m}$, with peak sizes at around $\sim 150 \mu \mathrm{m}$ (Fig. 3A). Sonication shifted the particle size distributions toward lower sizes, and the distributions appear bi-disperse, with a large particle size distribution between $\sim 0.6$ and $\sim 100 \mu \mathrm{m}$, and a smaller size distribution between $\sim 0.1$ and $\sim 0.6 \mu \mathrm{m}$. While the peak of the large size distribution depends on the oil concentration, at $1.5, \sim 2.0$ and $\sim 3.0 \mu \mathrm{m}$ for 20,35 and $50 \%$ respectively, that of the small size distributions remains independent of the oil concentration at $\sim 0.3 \mu \mathrm{m}$.

To compare the size of the different emulsions, the Sauter mean diameter $\left(D_{3,2}\right)$ is calculated from the particle size distributions (Fig. 3B). As expected, sonication, due to the high mechanical shear it exerts, reduced exponentially the particle size of the oil droplets [5]. It can be seen that the size of the pre-homogenised emulsions $(>10 \mu \mathrm{m})$ decreases exponentially at $\geq 5$ s sonication to a size at least 10 times smaller $(\sim 1 \mu \mathrm{m})$. Increasing further the sonication time does not result in a marked decrease in oil droplet size. For example in the case of the $20 \%$ emulsion sonication for 10 and 180 s resulted in a particle size of 0.7 and $\sim 0.4 \mu \mathrm{m}$, respectively. Emulsion with higher oil concentrations nearly do not show any change in particle size when the sonication time is $\geq 10 \mathrm{~s}$. The limiting size obtained at longer sonication times in the case of low oil concentrations depends on the viscosities of both the oil and aqueous phases as well as the nature (e.g. aggregated $v s$ individual proteins, and amphiphilicity) and the concentration of the stabiliser (i.e. the minimal droplet size is in theory related to the amount of the protein used and its full surface coverage value). However, in the case of the high oil concentrations, the limiting oil droplet size is mainly due to the gelation of the emulsion hindering further reduction in size as suggested above.

As mentioned in the introduction it was previously speculated, in the case of MPC85 stabilised emulsions obtained by high pressure homogenisation where emulsion aggregation was first observed [13], that aggregation occurs when the oil droplet-to-oil droplet distance is equal or smaller to the size of the MPC particle. Assuming a random packing of the oil droplets $\left(\phi_{c}=0.64\right)$ in the emulsion a simple equation allowing the calculation of the theoretical volume fraction $\left(\phi_{t h}\right)$ of the aggregated emulsion is given by [13]:

$\phi_{t h}=\phi_{c}\left(\frac{D}{D+\delta}\right)^{3}$

Where $D$ is the oil droplet diameter (measured here as $\mathrm{D}_{3,2}$ ) and $\delta$ ( $=117 \mathrm{~nm}$, the measured size of a WPC70 particle) is the oil droplet-todroplet distance.

To investigate the validity of Equation (1) emulsions containing 30 to $65 \%$ oil were sonicated at $2.5 \mathrm{~s}$ intervals and assessed visually for their state of aggregation. Once the emulsion starts to gel (aggregation threshold), no further sonication is applied, and the oil droplet particle size is measured. The particle size of the oil droplets $D_{3,2}$ at the gelation threshold is plotted as a function of the oil volume fraction in the Inset of Fig. 3B, where it can be seen that the size increases exponentially with the oil concentration. Knowing the particle size at a given oil concentration allows calculating the theoretical oil volume fraction $\phi_{t h}$ using Equation (1). The results of this exercise are reported in Fig. 4. Firstly at $\phi \leq 55 \%$, the calculated $\phi_{t h}$ (open symbols and dashed line) overestimate the oil volume fraction $\phi$ of the emulsion (dotted line) by approximately an extra $10 \%$, which is a large amount. To remedy to this,

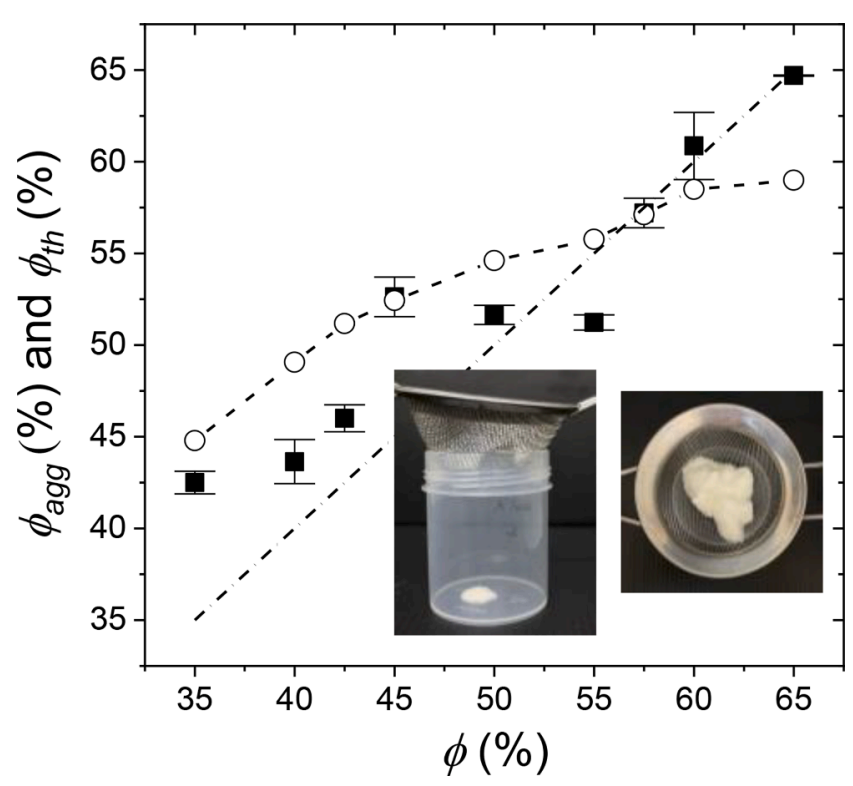

Fig. 4. Measured oil volume fraction in the aggregates $\left(\phi_{\text {agg }}\right)(\square)$ and calculated volume fraction using equation (1) $\left(\phi_{\text {agg }}\right)(\mathrm{o}$, with dashed line as a guide for the eye) as a function of the oil volume fraction $\phi$. The dashed-dot line is a linear $\mathrm{y}=\phi$ curve. Error bars correspond to standard deviations. Inset: photographs of emulsion made with $\phi=40 \%$ sonicated for $5 \mathrm{~s}$ being left in a sieve to separate the liquid phase. 
the volume fraction of the aggregates ( $\left.\phi_{a g g}\right)$ is measured by draining the emulsion's liquid phase, present in the emulsion voids observed by confocal microscopy, through a sieve (Inset photographs in Fig. 4). Despite this, the calculated $\phi_{t h}$ still overestimate $\phi_{a g g}$ (solid symbols). It is worth mentioning that for $45 \% \leq \phi \leq 55 \%$, $\phi_{\text {agg }}$ is nearly constant at around $50 \%$, which would indicate that the aggregated emulsion without voids is made of $\sim 50 \%$ oil.

At high $\phi(=65 \%)$ the calculated $\phi_{t h}$ is smaller than $\phi$. This could be due to the fact that Equation (1) is valid for emulsion with a volume fraction $\phi$ below the close packing $\left(\phi \leq \phi_{c}=0.64\right)$, since at higher $\phi$ the droplets are either expected to deform due to crowding or to exist under a non-random packing (for instance hexagonal packing for monodisperse emulsion is $\phi_{c}=0.74$ ). Further, taking into account the volume occupied by the MPC70 particles, it is possible that at high $\phi$ the oil droplets start to deform and lose their spherical shape, making Equation (1) not valid. Thus, in this work only at $\phi$ close to $\phi_{c}$ that the calculated $\phi_{t h}$ is in agreement with both $\phi$ and $\phi_{a g g}$. In addition to the reasons provided above for the failure of Equation (1), it can be also due to the emulsion polydispersity since this equation is derived for emulsions with monodisperse particle size distributions. Further, another assumption made is that the oil droplets are fully covered by a homogeneous layer made of spherical MPC70 particle with a size of $177 \mathrm{~nm}$. This is in reality not the case since MPC70, and other casein-based ingredients such as skim milk and other MPCs also contain whey proteins, mainly $\beta$-lactoglobulin and $\alpha$-lactalbumin, representing approximately $20 \%$ of the total proteins, as well as some free individual caseins [20-21]. Both the whey proteins and the free caseins are expected to compete for adsorption into the oil droplet interface, resulting in a non-homogeneous protein layer as previously observed by transmission electron microscopy in the case of milk fat globules sonicated in the presence of skim milk [22].

\section{Conclusions}

This work shows that it is possible to generate aggregated emulsion with high viscosity when the oil volume fraction used is $\geq 35 \%$ using a casein-based dairy ingredient such as MPC70. To verify this, we sonicated emulsions $(\phi=50 \%)$ prepared with skim milk powder and MPC85, both also casein-based ingredients, and this resulted in aggregated emulsions; while emulsions made by individual caseins, such as sodium caseinate, are known to not form aggregates. This confirms that emulsion aggregation is due to the bridging of the oil droplets by the casein micelles. This works highlights the advantages of US compared to other homogenisation methods to form instantaneously gelled emulsions; the use of US treatment will avoid for example the clogging of a high pressure homogeniser due to high viscosity. Further, the viscosity behaviour and the extent of aggregation can be tuned by varying the ultrasound energy density, which in the case of a semi-dilute emulsion (at around 35\%) can vary from a high viscosity pseudoplastic behaviour to a liquid behaviour with the increase in the US energy.

Because of the shortcoming of the simple theory (Equation (1)) used in this paper, we suggest in future works to use a dairy ingredient, such as micellar casein concentrate, which is made mainly of casein micelles to ensure that the oil droplet interface is homogenously covered. As for a theoretical approach, it will be interesting to explore if recent developments on Pickering emulsions [23] could be extended to these systems.

Finally, the findings of this study might find applications in the dairy foods with semi-solid textures, and in high oil emulsions, such as mayonnaises and salad dressings. Further it will be worth investigating if the replacement of MPC70 by some plant-based proteins, such as quinoa protein isolate $[24,25]$ and pea protein isolate, which are known to form aggregates, might yield emulsions with similar characteristics.

\section{CRediT authorship contribution statement}

Ruijia Zhang: Investigation, Formal analysis, Data curation. Lan
Luo: Investigation, Formal analysis, Data curation. Zhi Yang: Supervision, Conceptualization, Formal analysis, Data curation, Funding acquisition, Writing - review \& editing, Project administration. Muthupandian Ashokkumar: Writing - review \& editing. Yacine Hemar: Conceptualization, Formal analysis, Data curation, Supervision, Writing - original draft, Writing - review \& editing, Project administration.

\section{Declaration of Competing Interest}

The authors declare that they have no known competing financial interests or personal relationships that could have appeared to influence the work reported in this paper.

\section{Acknowledgements}

Zhi Yang would like to thank the financial support by the College of Sciences Massey University Research Fund (MURF) for Early Career Researchers.

\section{References}

[1] B. Abismail, J.P. Canselier, A.M. Wilhelm, H. Delmas, C. Gourdon, Emulsification by ultrasound: drop size distribution and stability, Ultrason. Sonochem. 6 (1-2) (1999) 75-83.

[2] J.P. Canselier, H. Delmas, A.M. Wilhelm, B. Abismail, Ultrasound emulsificationAn overview, J. Dispersion Sci. Technol. 23 (1-3) (2002) 333-349.

[3] L. Patil, P.R. Gogate, Ultrasound assisted synthesis of stable oil in milk emulsion: Study of operating parameters and scale-up aspects, Ultrason. Sonochem. 40 (2018) 135-146.

[4] A. Taha, E. Ahmed, A. Ismaiel, M. Ashokkumar, X. Xu, S. Pan, H. Hu, Ultrasonic emulsification: An overview on the preparation of different emulsifiers-stabilized emulsions, Trends Food Sci. Technol. 105 (2020) 363-377.

[5] W.u. Li, T.S.H. Leong, M. Ashokkumar, G.J.O. Martin, A study of the effectiveness and energy efficiency of ultrasonic emulsification, PCCP 20 (1) (2018) 86-96.

[6] M. Ashokkumar, R. Bhaskaracharya, S. Kentish, J. Lee, M. Palmer, B. Zisu, The ultrasonic processing of dairy products -An overview, Dairy, Science \& Technology. $90(2-3)(2010)$ 147-168.

[7] C. Holt, J.A. Carver, H. Ecroyd, D.C. Thorn, Invited review: Caseins and the casein micelle: Their biological functions, structures, and behavior in foods, J. Dairy Sci. 96 (2013) 6127-6146.

[8] D.S. Horne, Casein interactions: Casting light on the black boxes, the structure in dairy products, Int. Dairy J. 8 (1998) 171-177.

[9] C.G. de Kruif, T. Huppertz, V.S. Urban, A.V. Petukhov, Casein micelles and their internal structure, Adv. Colloid Interface Sci. 171-172 (2012) 36-52.

[10] A. Shanmugam, M. Ashokkumar, Ultrasonic preparation of stable flax seed oil emulsions in dairy systems - Physicochemical characterization, Food Hydrocolloids 39 (2014) 151-162.

[11] S. Anandan, M. Keerthiga, S. Vijaya, A.M. Asiri, V. Bogush, O. Krasulyaa, Physicochemical characterization of black seed oil-milk emulsions through ultrasonication, Ultrason. Sonochem. 38 (2017) 766-771.

[12] M. Silva, B. Zisu, J. Chandrapala, Stability of oil-water primary emulsions stabilised with varying levels of casein and whey proteins affected by highintensity ultrasound, Int. J. Food Sci. Technol. 56 (2) (2021) 897-908.

[13] Y. Hemar, C.E. Hall, H. Singh, Rheological properties of oil-in-water emulsions formed with milk protein concentrate, J. Texture Stud. 36 (3) (2005) 289-302.

[14] S.V. Crowley, B. Desautel, I. Gazi, A.L. Kelly, T. Huppertz, J.A. O'Mahony, Rehydration characteristics of milk protein concentrate powders, J. Food Eng. 149 (2015) 105-113.

[15] R.F. Contamine, A.M. Wilhelm, J. Berlan, H. Delmas, Power measurement in sonochemistry, Ultrason. Sonochem. 2 (1) (1995) S43-S47.

[16] A.R. Mackie, A.P. Gunning, P.J. Wilde, V.J. Morris, Orogenic displacement of protein from the oil/water interface, Langmuir 16 (5) (2000) 2242-2247.

[17] A.M. Mota da Silva, F. Souza Almeida, A.C. Kawazoe Sato, Functional characterization of commercial plant proteins and their application on stabilization of emulsions, J. Food Eng. 292 (2021), 110277.

[18] I. Dankar, A. Haddarah, F. El Omar, F. Sepulcre, M. Pujolà, Assessing the microstructural and rheological changes induced by food additives on potato puree, Food Chem. 240 (2018) 304-313.

[19] A. Tárrega, L. Durán, E. Costell, Flow behaviour of semi-solid dairy desserts, Effect of temperature, International Dairy Journal. 14 (4) (2004) 345-353.

[20] S.R. Euston, R.L. Hirst, Comparison of the concentration-dependent emulsifying properties of protein products containing aggregated and non-aggregated milk protein, Int. Dairy J. 9 (10) (1999) 693-701.

[21] Y. Xu, D. Liu, H. Yang, J. Zhang, X. Liu, J.M. Regenstein, Y. Hemar, P. Zhou, Effect of calcium sequestration by ion-exchange treatment on the dissociation of casein micelles in model milk protein concentrates, Food Hydrocolloids 60 (2016) 59-66.

[22] F.C. Nisa, Influence of low-frequency ultrasound homogenisation on the physicochemical properties of milk cream and milk fat-skim milk mixtures, 
(Doctoral thesis University of Auckland, Auckland New Zealand), 2019. Retrieved from.

[23] S.F. Velandia, P. Marchal, Cécile Lemaitre, Véronique Sadtler, T. Roques-Carmes, Evaluation of the repartition of the particles in Pickering emulsions in relation with their rheological properties, J. Colloid Interface Sci. 589 (2021) 286-297.

[24] R. Zhang, L. Cheng, L. Luo, Y. Hemar, Z. Yang, Formation and characterisation of high-internal-phase emulsions stabilised by high-pressure homogenised quinoa protein isolate, Colloids and Surfaces A: Physicochemi. Eng. Aspects 631 (2021), https://doi.org/10.1016/j.colsurfa.2021.127688.

[25] Z. Yang, L. de Campo, E.P. Gilbert, R. Knott, L. Cheng, B. Storer, X. Lin, L. Luo, S. Patole, Y. Hemar, Effect of $\mathrm{NaCl}$ and $\mathrm{CaCl}_{2}$ concentration on the rheological and structural characteristics of thermally-induced quinoa protein gels, Food Hydrocolloids 124 (2022), https://doi.org/10.1016/j.foodhyd.2021.107350. 


\section{University Library}

\section{- M M I N E R VA A gateway to Melbourne's research publications}

Minerva Access is the Institutional Repository of The University of Melbourne

Author/s:

Zhang, R;Luo, L;Yang, Z;Ashokkumar, M;Hemar, Y

Title:

Formation by high power ultrasound of aggregated emulsions stabilised with milk protein concentrate (MPC70).

Date:

2021-12-03

\section{Citation:}

Zhang, R., Luo, L., Yang, Z., Ashokkumar, M. \& Hemar, Y. (2021). Formation by high power ultrasound of aggregated emulsions stabilised with milk protein concentrate (MPC70).. Ultrason Sonochem, 81, pp.105852-. https://doi.org/10.1016/j.ultsonch.2021.105852.

Persistent Link:

http://hdl.handle.net/11343/296139

License:

CC BY-NC-ND 